\title{
Hypergeometric series and the Riemann zeta function
}

\author{
by
}

Wenchang Chu (Roma)

For infinite series related to the Riemann zeta function, De Doelder [4] established numerous interesting identities through evaluating improper integrals. Some of them are rederived in [2] by means of the Parseval identity for Fourier series. A couple of the most elegant examples may be reproduced as

$$
\begin{aligned}
\sum_{n=1}^{\infty}\left(\frac{1+\frac{1}{2}+\ldots+\frac{1}{n}}{n}\right)^{2} & =\frac{17 \pi^{4}}{360}=\frac{17}{4} \zeta(4), \\
\sum_{n=1}^{\infty}\left(\frac{1+\frac{1}{2}+\ldots+\frac{1}{n}}{n^{3}}\right) & =\frac{\pi^{4}}{72}=\frac{5}{4} \zeta(4),
\end{aligned}
$$

where the latter is due to Euler (1775). Other formulas and the related references may be found in Berndt [1, Ch. 9].

Since the first glance at the paper of Borweins [2], the author has been stimulated, by the beauty of these formulas, to search for a different way of dealing with such kind of infinite series. The present paper will show that the hypergeometric method can fulfill that purpose, which may be illustrated by the following simple example.

For complex parameters $\{x, c\}$, consider the binomial expansion

$$
(1+x)^{c}=\sum_{n=0}^{\infty}\left(\begin{array}{l}
c \\
n
\end{array}\right) x^{n}
$$

The coefficient of $c^{m+1}$ gives a general infinite series identity

$$
\frac{\ln ^{m+1}(1+x)}{(m+1) !}=\sum_{n=0}^{\infty}(-1)^{m+n} \frac{e_{m}(n)}{n+1} x^{n+1},
$$

1991 Mathematics Subject Classification: Primary 11M06; Secondary 33C20.

Key words and phrases: the Riemann zeta function, the harmonic numbers, hypergeometric series, the gamma function, symmetric functions. 
where $e_{m}(n)$ is the $m$ th elementary symmetric function in $\{1,1 / 2, \ldots, 1 / n\}$. Its specification, for $x=m=1$, leads us to a strange summation formula

$$
\frac{\ln ^{2} 2}{2}=\sum_{n=0}^{\infty}(-1)^{n}\left(\frac{1+\frac{1}{2}+\ldots+\frac{1}{n+1}}{n+2}\right)
$$

due to De Doelder [4, Eq. 25].

This method works for most of the hypergeometric summation theorems. With the notation from Slater [7], the generalized hypergeometric series (unilateral and bilateral) read as

$$
\begin{aligned}
{ }_{1+p} F_{q}\left[\begin{array}{r}
u_{0}, u_{1}, \ldots, u_{p} \\
v_{1}, \ldots, v_{q}
\end{array} ; z\right] & =\sum_{n=0}^{\infty} \frac{\left(u_{0}\right)_{n}\left(u_{1}\right)_{n} \ldots\left(u_{p}\right)_{n}}{n !\left(v_{1}\right)_{n} \ldots\left(v_{q}\right)_{n}} z^{n}, \\
{ }_{p} H_{q}\left[\begin{array}{c}
u_{1}, u_{2}, \ldots, u_{p} \\
v_{1}, v_{2}, \ldots, v_{q}
\end{array} z\right] & =\sum_{n=-\infty}^{\infty} \frac{\left(u_{1}\right)_{n}\left(u_{2}\right)_{n} \ldots\left(u_{p}\right)_{n}}{\left(v_{1}\right)_{n}\left(v_{2}\right)_{n} \ldots\left(v_{q}\right)_{n}} z^{n},
\end{aligned}
$$

where the shifted factorial is defined, for a complex number $w$, by

$$
(w)_{n}= \begin{cases}w(w+1) \ldots(w+n-1), & n=1,2, \ldots, \\ 1, & n=0, \\ (w-1)(w-2) \ldots(w+n), & n=-1,-2, \ldots\end{cases}
$$

On the one hand, the hypergeometric series may be expressed in terms of the partial sums of the Riemann zeta function through the symmetric functions generated by finite products (cf. [6])

$$
\prod_{k=1}^{n}(1+x / k)=1+x H_{n}+\frac{x^{2}}{2}\left(H_{n}^{2}-H_{n}^{\prime \prime}\right)+\ldots,
$$

$$
\begin{aligned}
& \prod_{k=1}^{n}(1-x / k)^{-1}=1+x H_{n}+\frac{x^{2}}{2}\left(H_{n}^{2}+H_{n}^{\prime \prime}\right)+\ldots, \\
& \prod_{k=1}^{n}\left(1+\frac{y}{2 k-1}\right)=1+y O_{n}+\frac{y^{2}}{2}\left(O_{n}^{2}-O_{n}^{\prime \prime}\right)+\ldots, \\
& \prod_{k=1}^{n}\left(1-\frac{y}{2 k-1}\right)^{-1}=1+y O_{n}+\frac{y^{2}}{2}\left(O_{n}^{2}+O_{n}^{\prime \prime}\right)+\ldots,
\end{aligned}
$$

whose first two terms

$$
\begin{aligned}
& H_{n}=\sum_{k=1}^{n} \frac{1}{k}, \quad O_{n}=\sum_{k=1}^{n} \frac{1}{2 k-1}, \\
& H_{n}^{\prime \prime}=\sum_{k=1}^{n} \frac{1}{k^{2}}, \quad O_{n}^{\prime \prime}=\sum_{k=1}^{n} \frac{1}{(2 k-1)^{2}}
\end{aligned}
$$

are closely related to the harmonic numbers. 
On the other hand, most of the hypergeometric summation theorems (cf. [3]) evaluate infinite series as a fraction of $\Gamma$-functions

$$
\Gamma\left[\begin{array}{c}
a, b, \ldots, c \\
A, B, \ldots, C
\end{array}\right]=\frac{\Gamma(a) \Gamma(b) \ldots \Gamma(c)}{\Gamma(A) \Gamma(B) \ldots \Gamma(C)},
$$

which may be expanded as multivariate formal power series. In fact, recalling, for the $\Gamma$-function (cf. [5]), the Weierstrass product

$$
\Gamma(z)=z^{-1} \prod_{n=1}^{\infty}\left\{(1+1 / n)^{z} /(1+z / n)\right\}
$$

and the logarithmic derivative

$$
\frac{\Gamma^{\prime}(z)}{\Gamma(z)}=-\gamma+\sum_{n=0}^{\infty} \frac{z-1}{(n+1)(n+z)}
$$

with the Euler constant

$$
\gamma=\lim _{n \rightarrow \infty}\left\{\sum_{k=1}^{n} \frac{1}{k}-\ln n\right\}
$$

we can derive the following expansions:

$$
\begin{aligned}
& \Gamma(1-z)=\exp \left\{\sum_{k=1}^{\infty} \frac{\sigma_{k}}{k} z^{k}\right\}, \\
& \Gamma\left(\frac{1}{2}-z\right)=\sqrt{\pi} \exp \left\{\sum_{k=1}^{\infty} \frac{\tau_{k}}{k} z^{k}\right\},
\end{aligned}
$$

where the Riemann zeta sequences $\left\{\sigma_{k}, \tau_{k}\right\}$ are defined by

$$
\begin{aligned}
& \sigma_{1}=\gamma, \quad \sigma_{m}=\zeta(m), \quad m=2,3, \ldots \\
& \tau_{1}=\gamma+2 \ln 2, \quad \tau_{m}=\left(2^{m}-1\right) \zeta(m), \quad m=2,3, \ldots
\end{aligned}
$$

For a specific hypergeometric summation theorem, both power series expansions mentioned above should coincide. The coefficients of a single monomial $x^{i} y^{j} z^{k} \ldots$ in both power series would result in a summation formula (trivial or non-trivial) similar to (0.1). By means of hypergeometric theorems, this approach will be extensively explored to establish several further infinite summation formulas. Some examples are

$$
\begin{aligned}
& \sum_{n=0}^{\infty} \frac{H_{n+1}^{\prime \prime}}{(n+1)^{2}}=\frac{7 \pi^{4}}{360}, \\
& \sum_{n=0}^{\infty} \frac{H_{2 n} H_{2 n+1}}{(2 n+1)^{2}}=\frac{\pi^{4}}{64},
\end{aligned}
$$




$$
\sum_{n=0}^{\infty} \frac{O_{n} O_{n+1}}{(2 n+1)^{2}}=\frac{\pi^{2}}{8} \ln ^{2} 2 .
$$

Because the hypergeometric method is quite mechanical and systematic, the tedious demonstration for summation formulas will not be presented in detail except for necessity. Instead, we will use a self-explanatory notation $\left[x^{i} y^{j} z^{k}\right]$ to indicate extracting the coefficients of the monomial $x^{i} y^{j} z^{k}$ from power series expansions. Throughout the paper, the Euler summation formulas

$$
\begin{array}{ll}
\sum_{n=1}^{\infty} \frac{1}{n^{2}}=\frac{\pi^{2}}{6}, & \sum_{n=0}^{\infty} \frac{(-1)^{n}}{2 n+1}=\frac{\pi}{4}, \\
\sum_{n=1}^{\infty} \frac{1}{n^{4}}=\frac{\pi^{4}}{90}, & \sum_{n=0}^{\infty} \frac{(-1)^{n}}{(2 n+1)^{3}}=\frac{\pi^{3}}{32},
\end{array}
$$

will frequently be applied without indication.

1. The Gauss summation theorem. Recall the Gauss theorem $[7$, p. 28]

$$
{ }_{2} F_{1}\left[\begin{array}{r}
x, y \\
1-z
\end{array} ; 1\right]=\Gamma\left[\begin{array}{l}
1-z, 1-x-y-z \\
1-x-z, 1-y-z
\end{array}\right] .
$$

We may reformulate it, by means of (0.6a), as a functional equation between two multivariate infinite series

$$
\begin{aligned}
\Gamma\left[\begin{array}{c}
1-z, 1-x-y-z \\
1-x-z, 1-y-z
\end{array}\right]=1+x y \sum_{n=0}^{\infty} \frac{\prod_{i=1}^{n}(1+x / i)(1+y / i)}{(n+1)^{2} \prod_{j=1}^{n+1}(1-z / j)} \\
=\exp \left\{\sum_{k=1}^{\infty} \frac{\sigma_{k}}{k}\left[z^{k}+(x+y+z)^{k}-(x+z)^{k}-(y+z)^{k}\right]\right\} \\
=\exp \left\{\sigma_{2} x y+\sigma_{3} x y(x+y+2 z)\right. \\
\left.+\sigma_{4} x y\left(x^{2}+y^{2}+3 x z+3 y z+3 z^{2}+\frac{3}{2} x y\right)+\ldots\right\},
\end{aligned}
$$

where the right hand side of the first equality may be expanded, via (0.3), as a power series. Then term-by-term comparison of the coefficients of the two power series results in an infinite number of summation formulas.

Example 1.1. Summation formulas [4, Eq. 2] related to $\zeta(3)$ :

$$
\begin{array}{ll}
{\left[x^{2} y\right]} & \sum_{n=0}^{\infty} \frac{H_{n}}{(n+1)^{2}}=\sigma_{3}=\zeta(3), \\
{[x y z]} & \sum_{n=0}^{\infty} \frac{H_{n+1}}{(n+1)^{2}}=2 \sigma_{3}=2 \zeta(3) .
\end{array}
$$


Example 1.2. Summation formulas [2, Eqs. 2-3] related to $\zeta(4)$ :

$$
\begin{aligned}
{\left[x^{2} y z\right] } & \sum_{n=0}^{\infty} \frac{H_{n} H_{n+1}}{(n+1)^{2}}=3 \sigma_{4}=\frac{\pi^{4}}{30}, \\
{\left[x^{2} y^{2}\right] } & \sum_{n=1}^{\infty} \frac{H_{n}^{2}}{(n+1)^{2}}=\frac{1}{2}\left(\sigma_{2}^{2}+3 \sigma_{4}\right)=\frac{11 \pi^{4}}{360}, \\
& \sum_{n=0}^{\infty} \frac{H_{n+1}^{2}}{(n+1)^{2}}=\frac{17 \sigma_{4}}{4}=\frac{17 \pi^{4}}{360} .
\end{aligned}
$$

ExAmple 1.3. Summation formulas [2, Eq. 4] and [4, Eq. 7] related to $\zeta(4):$

$$
\begin{aligned}
& \sum_{n=0}^{\infty} \frac{H_{n}}{(n+1)^{3}}=\frac{\sigma_{4}}{4}=\frac{\pi^{4}}{360}, \\
& \sum_{n=0}^{\infty} \frac{H_{n+1}}{(n+1)^{3}}=\frac{5 \sigma_{4}}{4}=\frac{\pi^{4}}{72} .
\end{aligned}
$$

EXAmPLE 1.4. More summation formulas related to $\zeta(4)$ :

$$
\begin{aligned}
& \sum_{n=0}^{\infty} \frac{H_{n}^{\prime \prime}}{(n+1)^{2}}=\frac{3}{4} \sigma_{4}=\frac{\pi^{4}}{120}, \\
& \sum_{n=0}^{\infty} \frac{H_{n+1}^{\prime \prime}}{(n+1)^{2}}=\frac{7}{4} \sigma_{4}=\frac{7 \pi^{4}}{360} .
\end{aligned}
$$

Sketch of proof. The coefficients of $x y$ in both formal power series expansions lead us to Euler's formula $\zeta(2)=\pi^{2} / 6$. In Example 1.2, the third formula follows from the previous two identities since

$$
\begin{aligned}
H_{n+1}^{2} & =\left(H_{n}+\frac{1}{n+1}\right)^{2}=H_{n}^{2}+\frac{2 H_{n}}{n+1}+\frac{1}{(n+1)^{2}} \\
& =H_{n}^{2}+2 H_{n}\left(H_{n+1}-H_{n}\right)+\frac{1}{(n+1)^{2}} \\
& =2 H_{n} H_{n+1}-H_{n}^{2}+\frac{1}{(n+1)^{2}} .
\end{aligned}
$$

Linear combinations of the identities of Example 1.2 yield the formulas in Example 1.3. Other two terms

$$
\begin{aligned}
{\left[x y z^{2}\right] } & \sum_{n=1}^{\infty} \frac{H_{n}^{2}+H_{n}^{\prime \prime}}{n^{2}}=6 \sigma_{4}=\frac{\pi^{4}}{15}, \\
{\left[x^{3} y\right] } & \sum_{n=0}^{\infty} \frac{H_{n}^{2}-H_{n}^{\prime \prime}}{(n+1)^{2}}=2 \sigma_{4}=\frac{\pi^{4}}{45}
\end{aligned}
$$

can be used to derive, without difficulty, the formulas in the last example. 
2. The Kummer summation theorem. The Kummer theorem [7, p. 51]

$$
{ }_{2} F_{1}\left[\begin{array}{c}
x, \quad y \\
1+x-y
\end{array} ;-1\right]=\Gamma\left[\begin{array}{l}
1+x / 2,1+x-y \\
1+x, 1+x / 2-y
\end{array}\right]
$$

may be restated, through (0.6a), as

$$
\begin{aligned}
& \Gamma\left[\begin{array}{l}
1+x / 2,1+x-y \\
1+x, 1+x / 2-y
\end{array}\right] \\
& =1-x y \sum_{n=0}^{\infty}(-1)^{n} \frac{\prod_{i=1}^{n}(1+x / i)(1+y / i)}{(n+1)^{2} \prod_{j=1}^{n+1}[1-(y-x) / j]} \\
& =\exp \left\{\sum_{k=1}^{\infty}(-1)^{k} \frac{\sigma_{k}}{k \cdot 2^{k}}\left[x^{k}+(2 x-2 y)^{k}-(2 x)^{k}-(x-2 y)^{k}\right]\right\} \\
& =\exp \left\{-\frac{\sigma_{2}}{2} x y+\frac{\sigma_{3}}{4} x y(3 x-2 y)+\frac{\sigma_{4}}{8} x y\left(9 x y-7 x^{2}-4 y^{2}\right)+\ldots\right\}
\end{aligned}
$$

whose power series expansion via (0.3) leads us to infinite series identities.

EXAmple 2.1. Alternating sums [4, Eq. 5] related to $\zeta(3)$ :

$$
\begin{aligned}
{\left[x y^{2}\right] \quad } & \sum_{n=0}^{\infty}(-1)^{n} \frac{H_{n}+H_{n+1}}{(n+1)^{2}}=\frac{\sigma_{3}}{2}=\frac{\zeta(3)}{2}, \\
& \sum_{n=0}^{\infty}(-1)^{n} \frac{H_{n}}{(n+1)^{2}}=\frac{-\sigma_{3}}{8}=\frac{-\zeta(3)}{8}, \\
& \sum_{n=0}^{\infty}(-1)^{n} \frac{H_{n+1}}{(n+1)^{2}}=\frac{5}{8} \sigma_{3}=\frac{5}{8} \zeta(3) .
\end{aligned}
$$

EXAMPLE 2.2. Alternating sums related to $\zeta(4)$ :

$$
\begin{aligned}
{\left[x^{3} y\right] \quad } & \sum_{n=0}^{\infty}(-1)^{n} \frac{\left(H_{n}-H_{n+1}\right)^{2}}{(n+1)^{2}}=\frac{7}{8} \sigma_{4}=\frac{7 \pi^{4}}{720}, \\
{\left[x y^{3}\right] \quad } & \sum_{n=0}^{\infty}(-1)^{n} \frac{\left(H_{n}+H_{n+1}\right)^{2}}{(n+1)^{2}}=\frac{\sigma_{4}}{8}=\frac{\pi^{4}}{720}, \\
& \sum_{n=0}^{\infty}(-1)^{n} \frac{H_{n}^{2}+H_{n+1}^{2}}{(n+1)^{2}}=\frac{\sigma_{4}}{2}=\frac{\pi^{4}}{180}, \\
& \sum_{n=0}^{\infty}(-1)^{n} \frac{H_{n} H_{n+1}}{(n+1)^{2}}=\frac{-3 \sigma_{4}}{16}=\frac{-\pi^{4}}{480} .
\end{aligned}
$$


EXAMPLE 2.3. Alternating sums related to $\zeta(4)$ :

$$
\sum_{n=0}^{\infty}(-1)^{n}\left\{\frac{2 H_{n+1}}{(n+1)^{3}}+\frac{H_{n+1}^{\prime \prime}}{(n+1)^{2}}\right\}=\frac{37 \sigma_{4}}{16}=\frac{37 \pi^{4}}{1440} .
$$

Sketch of proof. In Example 2.1, separating the numerator $\left\{H_{n}+\right.$ $\left.H_{n+1}\right\}$ of the summand in (2.1a) leads us to the other two formulas from the same example. For Example 2.2, the last two formulas are simply linear combinations of the first two identities. Finally, Example 2.3 follows directly from

$$
\left[x^{2} y^{2}\right] \quad \sum_{n=0}^{\infty}(-1)^{n} \frac{H_{n+1}^{2}-H_{n}^{2}+H_{n+1}^{\prime \prime}}{(n+1)^{2}}=\frac{\sigma_{2}^{2}+9 \sigma_{4}}{8}=\frac{23 \pi^{4}}{1440},
$$

which is quite strange, but less elegant.

\section{The Dougall-Dixon summation theorem}

A. The Dougall-Dixon theorem [7, p. 56]

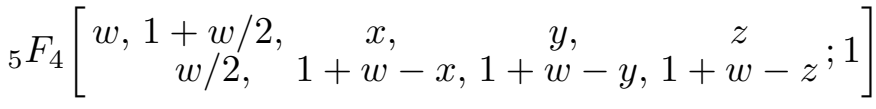

$$
\begin{aligned}
& =\Gamma\left[\begin{array}{l}
1+w-x, 1+w-y, 1+w-z, 1+w-x-y-z \\
1+w, 1+w-x-y, 1+w-x-z, 1+w-y-z
\end{array}\right]
\end{aligned}
$$

may be expressed, by means of (0.6a), as

$$
\begin{gathered}
\Gamma\left[\begin{array}{c}
1+w-x, 1+w-y, 1+w-z, 1+w-x-y-z \\
1+w, 1+w-x-y, 1+w-x-z, 1+w-y-z
\end{array}\right] \\
=1+x y z \sum_{n=0}^{\infty} \frac{w+2(n+1)}{(n+1)^{4}} \\
\quad \times \frac{\prod_{i=1}^{n}(1+w / i)(1+x / i)(1+y / i)(1+z / i)}{\prod_{j=1}^{n+1}[1+(w-x) / j][1+(w-y) / j][1+(w-z) / j]} \\
=\exp \left\{\sum _ { k = 1 } ^ { \infty } ( - 1 ) ^ { k } \frac { \sigma _ { k } } { k } \left[(w-x)^{k}+(w-y)^{k}+(w-z)^{k}+(w-x-y-z)^{k}\right.\right. \\
\left.\left.\quad-w^{k}-(w-x-y)^{k}-(w-x-z)^{k}-(w-y-z)^{k}\right]\right\} \\
=\exp \left\{2 \sigma_{3} x y z+3 \sigma_{4} x y z(x+y+z-2 w)+\ldots\right\}
\end{gathered}
$$

whose coefficient of $\left[x^{2} y z\right]$ recovers the results in Example 1.3. Another formula from this expansion may be stated as follows. 
EXAMPLE 3.1. An intriguing identity related to $\zeta(3)$ and $\zeta(6)$ :

$$
\left[(x y z)^{2}\right] \quad \sum_{n=0}^{\infty} \frac{\left(H_{n}+H_{n+1}\right)^{3}}{(n+1)^{3}}=\sigma_{3}^{2}+\frac{15}{2} \sigma_{6}=\zeta^{2}(3)+\frac{\pi^{6}}{126} .
$$

B. Replacing $z$ by $z+1 / 2$ in the Dougall-Dixon theorem, the resulting identity reads as

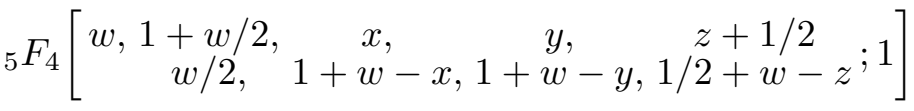

$$
\begin{aligned}
& =\Gamma\left[\begin{array}{l}
1+w-x, 1+w-y, 1 / 2+w-z, 1 / 2+w-x-y-z \\
1+w, 1+w-x-y, 1 / 2+w-x-z, 1 / 2+w-y-z
\end{array}\right]
\end{aligned}
$$

which may be restated as

$$
\begin{gathered}
\Gamma\left[\begin{array}{c}
1+w-x, 1+w-y, 1 / 2+w-z, 1 / 2+w-x-y-z] \\
1+w, 1+w-x-y, 1 / 2+w-x-z, 1 / 2+w-y-z
\end{array}\right] \\
=1+x y \sum_{n=0}^{\infty} \frac{w+2(n+1)}{(n+1)^{3}} \cdot \frac{\prod_{i=1}^{n}(1+w / i)(1+x / i)(1+y / i)}{\prod_{j=1}^{n+1}[1+(w-x) / j][1+(w-y) / j]} \\
\times \prod_{k=1}^{n+1} \frac{1+2 z /(2 k-1)}{1+2(w-z) /(2 k-1)} \\
=\exp \left\{\sum_{k=1}^{\infty}(-1)^{k} \frac{\sigma_{k}}{k}\left[(w-x)^{k}+(w-y)^{k}-w^{k}-(w-x-y)^{k}\right]\right\} \\
\quad \times \exp \left\{\sum _ { k = 1 } ^ { \infty } ( - 1 ) ^ { k } \frac { \tau _ { k } } { k } \left[(w-z)^{k}+(w-x-y-z)^{k}\right.\right. \\
=\exp \left\{2 \sigma_{2} x y+2 \sigma_{3} x y(3 x+3 y+7 z-6 w)+\ldots\right\} .
\end{gathered}
$$

The summation formulas obtained from its power series expansion are as follows.

EXAmple 3.2. Summation formulae [4, Eq. 15] related to $\zeta(3)$ :

$$
[x y z] \quad \sum_{n=1}^{\infty} \frac{O_{n}}{n^{2}}=\frac{7}{4} \sigma_{3}=\frac{7}{4} \zeta(3) .
$$

EXAMPLE 3.3. Summation formulas related to $\zeta(4)$ :

$$
\left[x^{2} y z\right] \quad \sum_{n=0}^{\infty} \frac{H_{n}+H_{n+1}}{(n+1)^{2}} O_{n+1}=\frac{45}{8} \sigma_{4}=\frac{\pi^{4}}{16},
$$




$$
\begin{array}{ll}
{\left[x y z^{2}\right]} & \sum_{n=0}^{\infty} \frac{O_{n+1}^{2}}{(n+1)^{2}}=\frac{45}{16} \sigma_{4}=\frac{\pi^{4}}{32}, \\
{\left[x^{2} y^{2}\right]} & \sum_{n=0}^{\infty} \frac{\left(H_{n}+H_{n+1}\right)^{2}}{(n+1)^{2}}=13 \sigma_{4}=\frac{13}{90} \pi^{4} .
\end{array}
$$

C. Replacing $w, x, y, z$ respectively by $1+w, x+1 / 2, y+1 / 2, z+1 / 2$ in the Dougall-Dixon theorem, the resulting evaluation reads as

$$
\begin{aligned}
& { }_{5} F_{4}\left[\begin{array}{c}
1+w,(3+w) / 2, \quad x+1 / 2, \\
(1+w) / 2,3 / 2+w-x, 3 / 2+w-y, 3 / 2+w-z
\end{array} ; 1\right] \\
& =\Gamma\left[\begin{array}{c}
3 / 2+w-x, 3 / 2+w-y, 3 / 2+w-z, 1 / 2+w-x-y-z \\
2+w, 1+w-x-y, 1+w-x-z, 1+w-y-z
\end{array}\right] .
\end{aligned}
$$

We may reformulate it as

$$
\begin{gathered}
\Gamma\left[\begin{array}{c}
1 / 2+w-x, 1 / 2+w-y, 1 / 2+w-z, 1 / 2+w-x-y-z] \\
1+w, 1+w-x-y, 1+w-x-z, 1+w-y-z
\end{array}\right] \\
=\sum_{n=0}^{\infty} \frac{w+1+2 n}{(n+1 / 2)^{3}} \cdot \frac{\prod_{i=1}^{n}\left(1+\frac{w}{i}\right)\left(1+\frac{2 x}{2 i-1}\right)\left(1+\frac{2 y}{2 i-1}\right)\left(1+\frac{2 z}{2 i-1}\right)}{\prod_{j=1}^{n+1}\left(1+\frac{2 w-2 x}{2 j-1}\right)\left(1+\frac{2 w-2 y}{2 j-1}\right)\left(1+\frac{2 w-2 z}{2 j-1}\right)} \\
=\pi^{2} \exp \left\{\sum _ { k = 1 } ^ { \infty } ( - 1 ) ^ { k } \frac { \tau _ { k } } { k } \left[(w-x)^{k}+(w-y)^{k}+(w-z)^{k}\right.\right. \\
\left.\left.+(w-x-y-z)^{k}\right]\right\} \\
\quad \times \exp \left\{-\sum_{k=1}^{\infty}(-1)^{k} \frac{\sigma_{k}}{k}\left[w^{k}+(w-x-y)^{k}+(w-x-z)^{k}\right.\right. \\
\left.\left.+(w-y-z)^{k}\right]\right\} \\
=\pi^{2} \exp \left\{4 \ln 2(x+y+z-2 w)+2 \sigma_{2}\left(x^{2}+y^{2}+z^{2}+2 w^{2}\right)\right. \\
\left.+2 \sigma_{2}(x y+x z+y z-2 w x-2 w y-2 w z)+\ldots\right\}
\end{gathered}
$$

which enables us to compute its coefficients through power series expansions.

EXAMPLE 3.4. A strange summation formulae:

$$
[x] \quad \sum_{n=0}^{\infty} \frac{O_{n}+O_{n+1}}{(2 n+1)^{2}}=\frac{\pi^{2}}{4} \ln 2 .
$$


EXAMPLE 3.5. Three strange summation formulas:

$$
\begin{aligned}
{[x y] } & \sum_{n=0}^{\infty} \frac{\left(O_{n}+O_{n+1}\right)^{2}}{(2 n+1)^{2}}=\frac{\pi^{2}}{96}\left(\pi^{2}+48 \ln ^{2} 2\right), \\
{\left[x^{2}\right] } & \sum_{n=0}^{\infty} \frac{O_{n}^{2}+O_{n+1}^{2}}{(2 n+1)^{2}}=\frac{\pi^{2}}{96}\left(\pi^{2}+24 \ln ^{2} 2\right), \\
& \sum_{n=0}^{\infty} \frac{O_{n} O_{n+1}}{(2 n+1)^{2}}=\frac{\pi^{2}}{8} \ln ^{2} 2 .
\end{aligned}
$$

Sketch of proof. The summation formula (3.3b) is due to De Doelder [4, Eq. 22] (cf. [2, Eq. 13]), and (3.5c) follows from the difference between the two previous identities in the last example.

\section{The Dixon-Kummer summation theorem}

A. When $z \rightarrow \infty$, the Dougall-Dixon theorem in the last section reduces to the Dixon-Kummer theorem [7, p. 56]

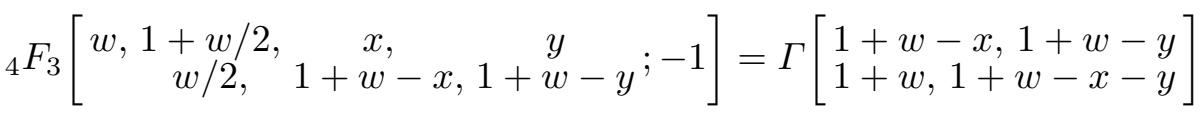

which may be expressed, through (0.6a), as

$$
\begin{aligned}
\Gamma\left[\begin{array}{c}
1+w-x, 1+w-y \\
1+w, 1+w-x-y
\end{array}\right] \\
=1-x y \sum_{n=0}^{\infty}(-1)^{n} \frac{w+2(n+1)}{(n+1)^{3}} \cdot \frac{\prod_{i=1}^{n}\left(1+\frac{w}{i}\right)\left(1+\frac{x}{i}\right)\left(1+\frac{y}{i}\right)}{\prod_{j=1}^{n+1}\left(1+\frac{w-x}{j}\right)\left(1+\frac{w-y}{j}\right)} \\
=\exp \left\{\sum_{k=1}^{\infty}(-1)^{k} \frac{\sigma_{k}}{k}\left[(w-x)^{k}+(w-y)^{k}-w^{k}-(w-x-y)^{k}\right]\right\} \\
=\exp \left\{\begin{array}{r}
-\sigma_{2} x y-\sigma_{3} x y(x+y-2 w) \\
\left.\quad-\sigma_{4} x y\left(3 w^{2}+x^{2}+y^{2}-3 w x-3 w y+\frac{3}{2} x y\right)+\ldots\right\} .
\end{array}\right.
\end{aligned}
$$

By means of (0.3), its power series expansion may be used to revisit the formulas displayed in Example 2.2.

B. Replacing $y$ by $y+1 / 2$ in the Dixon-Kummer theorem, the resulting evaluation reads as

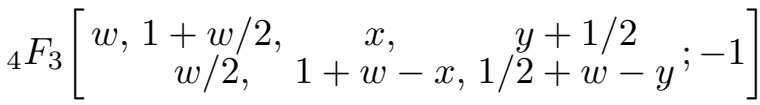

$$
\begin{aligned}
& =\Gamma\left[\begin{array}{l}
1+w-x, 1 / 2+w-y \\
1+w, 1 / 2+w-x-y
\end{array}\right]
\end{aligned}
$$


which may be restated as

$$
\begin{aligned}
& \Gamma\left[\begin{array}{c}
1+w-x, 1 / 2+w-y \\
1+w, 1 / 2+w-x-y
\end{array}\right] \\
& =1-x \sum_{n=0}^{\infty}(-1)^{n} \frac{w+2(n+1)}{(n+1)^{2}} \prod_{i=1}^{n+1} \frac{1+2 y /(2 i-1)}{\left(1+\frac{w-x}{i}\right)\left(1+\frac{2 w-2 y}{2 i-1}\right)} \\
& \quad \times \prod_{j=1}^{n}\left(1+\frac{w}{j}\right)\left(1+\frac{x}{j}\right) \\
& =\exp \left\{\sum_{k=1}^{\infty}(-1)^{k}\left[\frac{\sigma_{k}}{k}\left\{(w-x)^{k}-w^{k}\right\}+\frac{\tau_{k}}{k}\left\{(w-y)^{k}-(w-x-y)^{k}\right\}\right]\right\} \\
& =\exp \left\{-2 x \ln 2+\sigma_{2} x(2 w-x-3 y)-6 \sigma_{3} w^{2} x\right. \\
& \left.\quad-\sigma_{3} x\left(2 x^{2}+7 y^{2}-6 w x-14 w y+7 x y\right)+\ldots\right\} .
\end{aligned}
$$

The first few coefficients of its formal power series expansion via (0.3) yield the following summation formulas.

EXAMPLE 4.1. Alternating sums related to the harmonic series:

$$
\begin{array}{ll}
{[x] \quad \sum_{n=0}^{\infty} \frac{(-1)^{n}}{n+1}=\ln 2,} \\
{[x y] \quad \sum_{n=0}^{\infty}(-1)^{n} \frac{O_{n+1}}{n+1}=\frac{3}{8} \sigma_{2}=\frac{\pi^{2}}{16} .}
\end{array}
$$

EXAMPLE 4.2. Alternating sums related to the harmonic series:

$$
\begin{aligned}
{\left[x^{2}\right] \quad } & \sum_{n=0}^{\infty}(-1)^{n} \frac{H_{n}+H_{n+1}}{n+1}=\frac{1}{2}\left(\sigma_{2}-2 \ln ^{2} 2\right)=\frac{\pi^{2}}{12}-\ln ^{2} 2, \\
& \sum_{n=0}^{\infty}(-1)^{n} \frac{H_{n}}{n+1}=-\frac{\ln ^{2} 2}{2}, \\
& \sum_{n=0}^{\infty}(-1)^{n} \frac{H_{n+1}}{n+1}=\frac{\pi^{2}}{12}-\frac{\ln ^{2} 2}{2} .
\end{aligned}
$$

EXAMPLE 4.3. Alternating sums related to the harmonic series:

$$
\begin{array}{ll}
{\left[x y^{2}\right]} & \sum_{n=0}^{\infty}(-1)^{n} \frac{O_{n+1}^{2}}{n+1}=\frac{7}{16} \sigma_{3}=\frac{7}{16} \zeta(3) \quad[4, \text { Eq. 21] } \\
{\left[x^{2} y\right]} & \sum_{n=0}^{\infty}(-1)^{n} \frac{H_{n}+H_{n+1}}{n+1} O_{n+1}=\frac{1}{8}\left(7 \zeta(3)-\pi^{2} \ln 2\right) .
\end{array}
$$


EXAMPLE 4.4. Alternating sums related to the harmonic series:

$$
\begin{aligned}
{\left[x^{3}\right] \quad } & \sum_{n=0}^{\infty}(-1)^{n} \frac{H_{n}^{2}+H_{n+1}^{2}}{n+1}=\zeta(3)+\frac{2}{3} \ln ^{3} 2-\frac{\pi^{2}}{6} \ln 2, \\
& \sum_{n=0}^{\infty}(-1)^{n} \frac{H_{n}^{2}}{n+1}=\frac{1}{4} \zeta(3)+\frac{1}{3} \ln ^{3} 2-\frac{\pi^{2}}{12} \ln 2, \\
& \sum_{n=0}^{\infty}(-1)^{n} \frac{H_{n+1}^{2}}{n+1}=\frac{3}{4} \zeta(3)+\frac{1}{3} \ln ^{3} 2-\frac{\pi^{2}}{12} \ln 2 .
\end{aligned}
$$

C. Replacing $w, x, y$ respectively by $1+w, x+1 / 2, y+1 / 2$ in the Dixon-Kummer theorem, the evaluation reads as

$$
\begin{array}{r}
{ }_{4} F_{3}\left[\begin{array}{r}
1+w,(3+w) / 2, \quad x+1 / 2, \\
(1+w) / 2,3 / 2+w-x, 3 / 2+w-y
\end{array} ;-1\right] \\
=\Gamma\left[\begin{array}{c}
3 / 2+w-x, 3 / 2+w-y \\
2+w, 1+w-x-y
\end{array}\right]
\end{array}
$$

which may be reformulated, through (0.6a) and (0.6b), as

$$
\begin{aligned}
& \Gamma\left[\begin{array}{c}
1 / 2+w-x, 1 / 2+w-y \\
1+w, 1+w-x-y
\end{array}\right] \\
& =\sum_{n=0}^{\infty}(-1)^{n} \frac{w+1+2 n}{\left(n+\frac{1}{2}\right)^{2}} \cdot \frac{\prod_{i=1}^{n}\left(1+\frac{w}{i}\right)\left(1+\frac{2 x}{2 i-1}\right)\left(1+\frac{2 y}{2 i-1}\right)}{\prod_{j=1}^{n+1}\left(1+\frac{2 w-2 x}{2 j-1}\right)\left(1+\frac{2 w-2 y}{2 j-1}\right)} \\
& =\pi \exp \left\{\sum _ { k = 1 } ^ { \infty } ( - 1 ) ^ { k } \left[\frac{\tau_{k}}{k}\left\{(w-x)^{k}+(w-y)^{k}\right\}\right.\right. \\
& \left.\left.-\frac{\sigma_{k}}{k}\left\{w^{k}+(w-x-y)^{k}\right\}\right]\right\} \\
& =\pi \exp \{2 \ln 2(x+y-2 w) \\
& \left.+\sigma_{2}\left(2 w^{2}+x^{2}+y^{2}-x y-2 w x-2 w y\right)+\ldots\right\} .
\end{aligned}
$$

Its power series expansion via (0.3) yields infinite series identities.

EXAMPLE 4.5. Alternating sums related to the harmonic series:

$$
\begin{aligned}
{[x] \quad } & \sum_{n=0}^{\infty}(-1)^{n} \frac{O_{n}+O_{n+1}}{2 n+1}=\frac{\pi}{4} \ln 2, \\
& \sum_{n=0}^{\infty}(-1)^{n} \frac{O_{n}}{2 n+1}=\frac{\pi}{8} \ln 2-\frac{\pi^{2}}{24},
\end{aligned}
$$




$$
\sum_{n=0}^{\infty}(-1)^{n} \frac{O_{n+1}}{2 n+1}=\frac{\pi}{8} \ln 2+\frac{\pi^{2}}{24} .
$$

EXAMPLE 4.6. Alternating sums related to the harmonic series:

$$
\begin{aligned}
& {[x y] \quad \sum_{n=0}^{\infty}(-1)^{n} \frac{\left(O_{n}+O_{n+1}\right)^{2}}{2 n+1}=\frac{\pi}{16}\left(4 \ln ^{2} 2-\sigma_{2}\right)} \\
& =\frac{\pi}{96}\left(24 \ln ^{2} 2-\pi^{2}\right) \text {, } \\
& {\left[x^{2}\right] \quad \sum_{n=0}^{\infty}(-1)^{n} \frac{O_{n}^{2}+O_{n+1}^{2}}{2 n+1}=\frac{\pi}{16}\left(2 \ln ^{2} 2+\sigma_{2}\right)} \\
& =\frac{\pi}{96}\left(12 \ln ^{2} 2+\pi^{2}\right) \text {, } \\
& \sum_{n=0}^{\infty}(-1)^{n} \frac{O_{n} O_{n+1}}{2 n+1}=\frac{\pi}{96}\left(6 \ln ^{2} 2-\pi^{2}\right) .
\end{aligned}
$$

Sketch of proof. The formula (4.1a) in Example 4.1 may be traced back to Newton and Leibniz, and obtained from classical analysis. In Examples 4.2, 4.4 and 4.5, the last two formulas are trivially derived from the first, respectively, by separating the corresponding summands. Among these identities, the identity (4.4b) in Example 4.4 gives a corrected version of [4, Eq. 12]. The difference between the first two identities in Example 4.6 results in the last formula in the same example.

\section{Concluding remarks}

A. For the bilateral hypergeometric series, we have two general summation theorems due to Dougall [7, pp. 181-182]:

$$
{ }_{2} H_{2}\left[\begin{array}{c}
u, v \\
1-x, 1-y
\end{array} ; 1\right]=\Gamma\left[\begin{array}{c}
1-u, 1-v, 1-x, 1-y, 1-u-v-x-y \\
1-u-x, 1-u-y, 1-v-x, 1-v-y
\end{array}\right]
$$

and

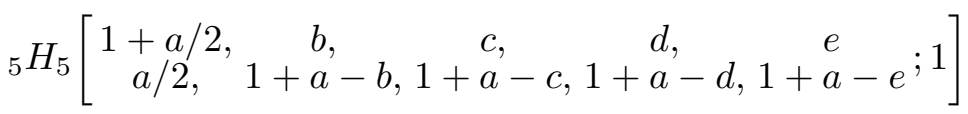

$$
\begin{aligned}
& =\Gamma\left[\begin{array}{c}
1+a-b, 1+a-c, 1+a-d, 1+a-e \\
1+a, 1+a-b-c, 1+a-b-d, 1+a-b-e
\end{array}\right] \\
& \times \Gamma\left[\begin{array}{c}
1-b, 1-c, 1-d, 1-e, 1+2 a-b-c-d-e \\
1-a, 1+a-c-d, 1+a-c-e, 1+a-d-e
\end{array}\right],
\end{aligned}
$$

which may be used to recover the formulas of Examples 1.2, 2.2, 3.4 and 3.5 , respectively. The only result that we find through bilateral summation 
theorem and which is essentially different from those given in the preceding sections, may be stated as

$$
\sum_{n=0}^{\infty}(-1)^{n}\left\{\frac{O_{n}^{\prime \prime}+O_{n+1}^{\prime \prime}}{(2 n+1)^{2}}+2 \frac{O_{n}+O_{n+1}}{(2 n+1)^{3}}\right\}=\frac{\pi^{3}}{8} \ln 2
$$

which somehow resembles the formula in Example 2.3.

B. Combining Examples 1.1 and 2.1, we get four additional summation formulas related to $\zeta(3)$ with even and odd summation indices:

$$
\begin{aligned}
& \sum_{n=1}^{\infty} \frac{H_{2 n-1}}{(2 n)^{2}}=\frac{9}{16} \zeta(3), \\
& \sum_{n=1}^{\infty} \frac{H_{2 n}}{(2 n)^{2}}=\frac{11}{16} \zeta(3), \\
& \sum_{n=0}^{\infty} \frac{H_{2 n}}{(2 n+1)^{2}}=\frac{7}{16} \zeta(3), \\
& \sum_{n=0}^{\infty} \frac{H_{2 n+1}}{(2 n+1)^{2}}=\frac{21}{16} \zeta(3) .
\end{aligned}
$$

Similarly, we may obtain from Examples 1.2 and 2.2 the following summation formulas related to $\zeta(4)$ :

$$
\begin{aligned}
& \sum_{n=1}^{\infty} \frac{H_{2 n} H_{2 n-1}}{(2 n)^{2}}=\frac{17}{960} \pi^{4}, \\
& \sum_{n=0}^{\infty} \frac{H_{2 n} H_{2 n+1}}{(2 n+1)^{2}}=\frac{\pi^{4}}{64}, \\
& \sum_{n=1}^{\infty} \frac{H_{2 n}^{2}+H_{2 n-1}^{2}}{(2 n)^{2}}=\frac{13}{360} \pi^{4}, \\
& \sum_{n=0}^{\infty} \frac{H_{2 n}^{2}+H_{2 n+1}^{2}}{(2 n+1)^{2}}=\frac{\pi^{4}}{24}, \\
& \sum_{n=1}^{\infty} \frac{\left(H_{2 n}+H_{2 n-1}\right)^{2}}{(2 n)^{2}}=\frac{103}{1440} \pi^{4}, \\
& \sum_{n=0}^{\infty} \frac{\left(H_{2 n}+H_{2 n+1}\right)^{2}}{(2 n+1)^{2}}=\frac{7}{96} \pi^{4} .
\end{aligned}
$$

C. Finally, from the power series expansions of the $\Gamma$-function we have

$$
\frac{\Gamma(z+\varepsilon)}{\Gamma(z)} \approx 1+\varepsilon \psi(z)+\frac{\varepsilon^{2}}{2}\left\{\psi^{2}(z)+\sum_{k \geq 0} \frac{1}{(k+z)^{2}}\right\},
$$




$$
\frac{\Gamma(z)}{\Gamma(z-\varepsilon)} \approx 1+\varepsilon \psi(z)+\frac{\varepsilon^{2}}{2}\left\{\psi^{2}(z)-\sum_{k \geq 0} \frac{1}{(k+z)^{2}}\right\},
$$

provided that $\varepsilon$ is an infinitesimal and the $\psi$-function is the logarithmic derivative of the $\Gamma$-function defined by (0.5).

Then the Gauss summation theorem stated in the first section may be expressed as the following infinite series transformation:

$$
\begin{aligned}
\sum_{n=0}^{\infty} \frac{n !(1+x)_{n}}{(n+1) !(1+y)_{n+1}} & =\lim _{\varepsilon \rightarrow 0} \frac{1}{\varepsilon x} \sum_{n>0} \frac{(\varepsilon)_{n}(x)_{n}}{n !(1+y)_{n}} \\
& =\lim _{\varepsilon \rightarrow 0} \frac{1}{\varepsilon x}\left\{-1+\Gamma\left[\begin{array}{l}
1+y, 1-\varepsilon-x+y \\
1-\varepsilon+y, 1-x+y
\end{array}\right]\right\} \\
& =\frac{1}{x}\{\psi(1+y)-\psi(1-x+y)\} \\
& =\sum_{k=1}^{\infty} \frac{1}{(k+y)(k-x+y)}
\end{aligned}
$$

whose reformulation

$$
\sum_{n=0}^{\infty} \frac{\prod_{k=1}^{n}(1+x / k)}{(n+1)^{2} \prod_{k=1}^{n+1}(1+y / k)}=\sum_{k=1}^{\infty} \frac{1}{(k+y)(k-x+y)}
$$

leads us, through the coefficient of $\left[x^{p} y^{q}\right]$, to a general summation formula

$$
\sum_{n=0}^{\infty} \frac{e_{p}(n) h_{q}(n+1)}{(n+1)^{2}}=\left(\begin{array}{c}
p+q+1 \\
p+1
\end{array}\right) \zeta(2+p+q)
$$

where $e_{k}(n)$ and $h_{k}(n)$ are respectively the elementary and complete symmetric functions of degree $k$ in $\{1,1 / 2, \ldots, 1 / n\}$. It contains most of the formulas in the first section as special cases.

Remark. By means of other hypergeometric summation theorems (cf. [3]) and formal power series expansions, it should be possible to derive further infinite series identities for $H_{n}$ and $O_{n}$. The interested reader is encouraged to pursue this direction.

\section{References}

[1] B. C. Berndt, Ramanujan's Notebooks, Part I, Springer, New York, 1985.

[2] D. Borwein and J. M. Borwein, On an intriguing integral and some series related to $\zeta(4)$, Proc. Amer. Math. Soc. 123 (1995), 1191-1198.

[3] W. Chu, Inversion techniques and combinatorial identities: A quick introduction to hypergeometric evaluations, in: Runs and Patterns in Probability: Selected Papers, A. P. Godbole and S. G. Papastavridis (eds.), Math. Appl. 283, Kluwer, Dordrecht, 1994, 31-57. 
[4] P. J. De Doelder, On some series containing $\psi(x)-\psi(y)$ and $(\psi(x)-\psi(y))^{2}$ for certain values of $x$ and $y$, J. Comput. Appl. Math. 37 (1991), 125-141.

[5] Y. L. Luke, The Special Functions and Their Approximations, Academic Press, London, 1969.

[6] I. G. Macdonald, Symmetric Functions and Hall Polynomials, Oxford Univ. Press, London, 1979.

[7] L. J. Slater, Generalized Hypergeometric Functions, Cambridge Univ. Press, Cambridge, 1966.

Istituto di Matematica "Guido Castelnuovo"

Università degli Studi di Roma "La Sapienza"

00185 Roma, Italy

E-mail: wenchang@mat.uniroma1.it

Received on 2.4.1996

and in revised form on 25.3.1997 